\title{
Cancer-associated fibroblasts support bone tropic metastasis by acting as coordinators between the tumor microenvironment and bone matrix in breast cancer
}

\author{
Minireview
}

\begin{abstract}
Hualing LI $1^{1,2,3, *}$, Xueguang LIN ${ }^{1,2, *}$, Di YANG ${ }^{1,2, *}$, Zhangyue CHEN ${ }^{1,2}$, Xiaotong WANG ${ }^{1,2}$, Fati $\mathrm{RE}^{4}$, Junjie WEi' ${ }^{1}$, Juping CHEN $^{1,2,3, *}$
${ }^{1}$ Institute of Translational Medicine, Medical College, Yangzhou University, Yangzhou, China; ${ }^{2}$ Jiangsu Key Laboratory of Experimental and Translational Non-coding RNA Research, Yangzhou University, Yangzhou, China; ${ }^{3} J i a n g s u$ Co-innovation Centre for Prevention and Control of Important Animal Infectious Diseases and Zoonoses, Yangzhou University, Yangzhou, China; ${ }^{4}$ Jiangsu Key Laboratory of Zoonosis, Yangzhou University, Yangzhou, China
\end{abstract}

*Correspondence: hlli@yzu.edu.cn \#Contributed equally to this work.

Received September 5, 2020 / Accepted October 27, 2020

\begin{abstract}
Bone is a common site of metastasis for various types of cancer cells, including breast cancer, and the consequent skeleton-related events observed in patients are severe and often fatal. Currently, it is widely accepted that cancer-associated fibroblasts (CAFs) confer a metastasis-promoting property to breast cancer cells. Furthermore, clinical observations suggest that CAFs mediate the bone tropism of metastatic breast cancer cells. Therefore, a deeper understanding of the mechanism by which CAFs are involved in the bone-tropic metastasis of breast cancer can facilitate the study of the novel and effective therapeutic drugs for the corresponding targets. In this review, we focused on the coordinator role of CAFs in remolding breast cancer cells and remodeling the bone marrow during metastasis. We discussed the potential roles of the CXCL12/ CXCR4 axis, the CAFs-CSCs reinforcing loop, and exosomes in this malignant process. In summary, in agreement with Paget's theory, CAFs play a pivotal role in bone colonization by breast cancer cells by providing a "fertile soil" for the "selected seeds" by influencing tumor-intrinsic characteristics and microenvironment (ME).
\end{abstract}

Key words: CAFs, breast cancer, bone metastasis, microenvironment, CXCL12, cancer stem cells, exosome, microRNA

Breast cancer is the most frequently diagnosed cancer among women and its treatment options are still far from sufficient. An important reason for treatment failure is the propensity of breast cancer for metastasizing to the skeletal system. In $70-80 \%$ of patients with advanced disease, bone metastasis eventually occurs. Breast cancer is a heterogeneous entity with a highly complicated tumor-associated microenvironment (ME), which consists of numerous stromal components, the extracellular matrix (ECM), and cells, such as cancer-associated fibroblasts (CAFs), immune-inflammatory cells, mesenchymal stem cells (MSCs), and endothelial cells $[1,2]$. Among these cells, CAFs are the major cellular type, and they are concomitantly involved in many steps of tumor progression from desmoplasia caused by excessive production of ECM in the primary tumor to metastasis outgrowth in secondary sites [3]. Studies have shown that the abundance of CAFs in tumor tissues is closely correlated with bone metastasis [3]. CAF-rich solid tumors, such as breast cancer, hepatocellular carcinoma, and prostate cancer, tend to have a higher selectivity for bone during metastases.

The key questions are whether specific factors can explain the relationship between CAFs and the tendency of cancer to metastasize to bone and whether the mechanism leads to an orchestrated process between tumor cells and bone ME. Factors highly expressed in the bone marrow, such as C-X-C motif chemokine 12 (CXCL12), glycosaminoglycan hyaluronic acid (HA), and osteopontin (OPN), are involved in the interactions among breast cancer, stromal cells, and the skeletal system that are necessary for the development of distant metastasis. For the tumor cells themselves, tumor 
heterogeneity is thought to rely on a distinct subset of tumor cells that have the ability to promote tumor growth. These cells are referred to as cancer stem cells (CSCs) and represent the true seeder cells in the metastasis process.

The role of exosomes is another key factor involved in the bone tropism of breast cancer metastasis because they are involved in establishing communication among epithelial cells, stromal cells in tumor beds, and pre-metastatic niches (PMNs). Among the messengers carried by exosomes, microRNA (miRNA) is a specific class of posttranscriptional modulators that can target those important routes. Specific miRNAs can cause an imbalance between the osteoblasts and osteoclasts that are involved in breast cancer bone metastasis. In this article, the characteristics of CAFs in breast cancer and their comprehensive role in modulating tumors and distant metastasis niches are reviewed with an emphasis on the coordination and incoordination that occur in the bone because these processes simultaneously alter both the "seed" and "soil".

\section{Origins and functions of CAFs}

CAFs are key stromal cells that play a dominant role in cancer pathogenesis. Complex network interactions could be established between CAFs and tumor cells through direct and rapid interactions with tumor cells. These interactions have been proposed to potentiate tumor cell initiation, proliferation, differentiation, tumor-related angiogenesis, metabolic pathways, and immune regulation [4]. Although some cancer therapy strategies are to target CAFs, their precise origin is still controversial. The prevailing view is that CAFs are fibroblasts that become activated from the quiescent state via stimulation with cytokines or other factors originating from tumor cells.

Activated fibroblasts can be identified by their expression of a-smooth muscle actin (aSMA), S100A4 (also known as fibroblast-specific protein 1), vimentin, fibroblast activation protein (FAP), and platelet-derived growth factor (PDGFR- $\alpha, \beta$ ) [5]. However, none of these markers is completely representative due to the heterogeneity of CAFs. Emerging evidence has suggested that CAFs develop from a variety of cells in addition to residential fibroblasts, including bone marrow-derived MSCs, stellate cells, adipose-derived mesenchymal stem cells (ADSCs), and monocytes (Figure 1). Moreover, recent studies have shown that CAFs are derived predominantly from local fibroblasts as well as a subpopulation of myofibroblasts in cancer, which are converted from circulating bone marrow-derived stem cells (BMSCs) [6]

Therefore, it is not surprising that the formation of several subsets with diverse cellular origins results in the heterogeneity and complexity of CAFs. For example, the transition of human adipose tissue-derived stem cells (hASCs) towards CAF-like cells has been demonstrated to occur under the influence of cancer-derived transforming growth factor (TGF $\beta 1$ ). These altered hASCs can enhance breast cancer cell aggressiveness in vitro by promoting the secretion of increased levels of CXCL12 and C-C motif chemokine ligand 5 (CCL5) [7]. Furthermore, studies have found that BMSC-derived CAFs exhibit a stronger angiogenic effect than colonized fibroblast-derived CAFs, while the latter has a

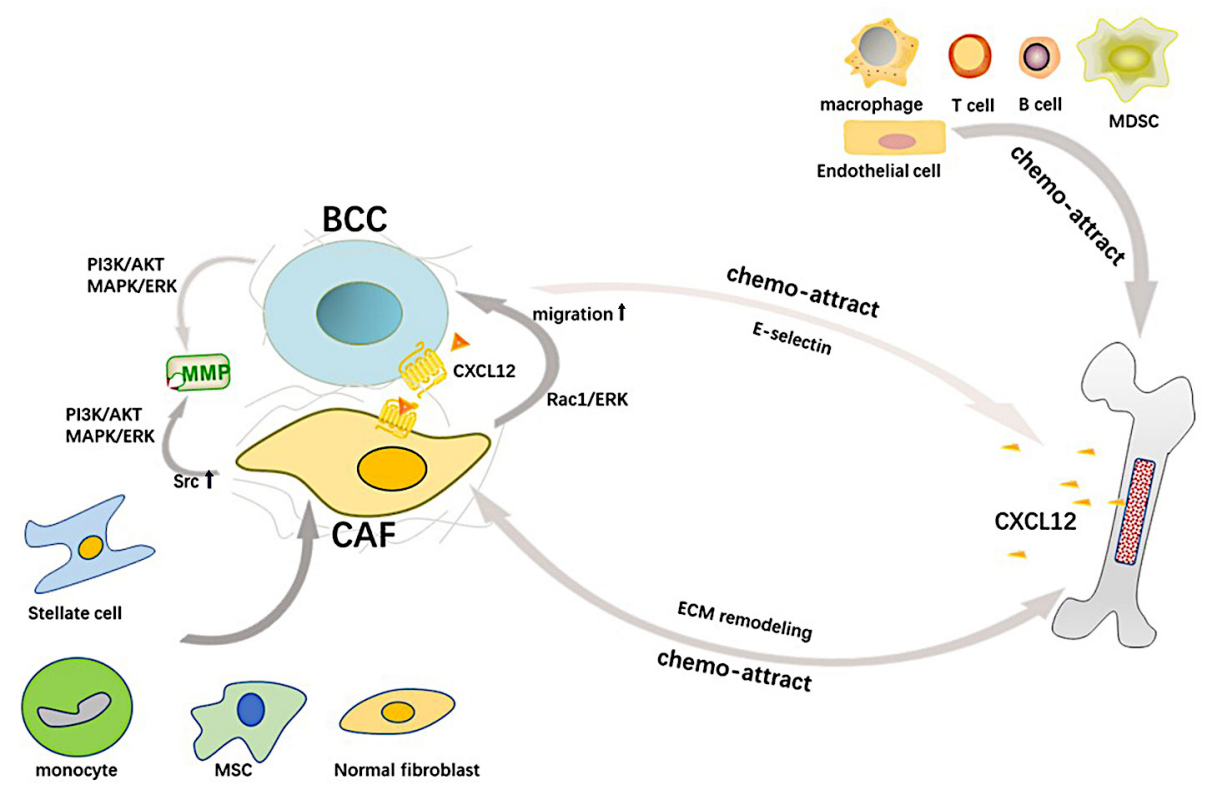

Figure 1. Role of CXCL12 in bone tropic metastasis of breast cancer and possible sources of CAF. Normal stromal cells are activated in situ via stimuli caused by damage. Activated normal fibroblasts account for the majority of CAFs, and the remnants are primarily generated from differentiated and migrated precursors from bone marrow. Here, CXCL12 mainly shows three important functions: affect the direction of tumor migration, change some characteristics of tumor cells, and participate in bone matrix remodeling. 
more obvious role in recruiting macrophages [8]. Therefore, in different types of solid tumors, the differences in biological characteristics, including invasiveness and metastatic tropism, are determined by the tumor stroma, which is composed of CAFs from different cell populations.

\section{Bone is the preferred metastatic niche for breast cancer}

Except for triple-negative breast cancer, which has a higher likelihood of visceral metastasis than bone metastasis, breast cancers, especially luminal breast cancer, often target bone as their first metastatic site. Tumor-associated complications, including severe pain, pathologic fracture, hypercalcemia, and spinal cord compression resulting from subsequent skeleton-related events (SREs), are important reasons underlying the low quality of life and high mortality among patients with advanced breast cancer.

In 1889, Paget proposed the "seed and soil" theory that suggests that tumor metastasis requires the participation of both internal and external factors. In breast cancer, the important role of bone as a "fertile soil" for the growth of breast cancer cells ("seeds") could be inferred from its propensity for bone metastasis. The characteristics that make bone suitable for the growth and colonization of breast cancer cells may be a result of the effects of multiple factors and corresponding receptors, including CXCL12, insulin-like growth factor 1 (IGF-1), hormone receptors, and parathyroid hormone-related protein $(\mathrm{PTHr})$, in the bone ME that are involved in the formation and resorption processes in bone tissue. Additionally, factors intrinsic to breast tumors could determine their bone metastatic potential because hormone receptor-positive luminal breast cancer has a more pronounced bone metastasis tendency than triple-negative breast cancer (TNBC) [9]. In addition, the phenotypic changes of the "seeds", such as the epithelialmesenchymal transition (EMT), mesenchymal-epithelial transition (MET), and osteomimicry, also affect this process [10]. During the progression of breast cancer, other cells are directly involved in the multiple metastatic steps of cancer cells. In summary, the two-way selection of "seed" and "soil" leads to the occurrence of bone metastases in breast cancer. In general, transient spatial coincidences and paracrine signaling between the breast cancer cells and the metastatic niche have been given more attention with respect to the important role of CAFs in the process of bone tropism in breast cancer metastasis.

\section{CAF-derived CXCL12 affects the recruitment, growth, and selection of breast cancer cells}

Roles of the CXCL12-CXCR4 axis in the recruitment and growth of breast cancer cells during the metastatic process. CXCL12 (also known as SDF1 $\alpha$ ) is widely expressed in the stromal fibroblasts of some tissues and organs, including lymph nodes, bone marrow, liver, lung, and especially CAFs [11]. On the one hand, the activation of the CXCL12-CXCR4 (C-X-C motif chemokine receptor 4) signaling pathway exhibits a variety of pro-metastasis properties, including chemotaxis, which plays a key role in the cell migration involved in lymphocyte homing, tumor cell metastasis, and endothelial cell migration. On the other hand, CXCR4, which is rarely expressed in normal tissues and closely related to tumorigenesis, is highly expressed in tumor cells and CAFs. As a result, the overexpression of CXCR4 on the surface of both breast cancer cells and CAFs as well as the massive amounts of soluble CXCL12 produced from the bone marrow act together to promote bone metastasis in breast cancer.

In addition to recruiting breast cancer cells and CAFs based on the differences in the expression gradients of the CXCL12 and CXCR4 chemokines between tumor cells and bone marrow [12], chemotaxis also enables bone marrow to recruit hematopoietic stem and progenitor cells (HSPCs), endothelial cells, myeloid-derived suppressor cells (MDSCs), lymphocytes, and monocytes to the bone ME, where they play a crucial role in constructing the suitable "soil" for metastatic breast tumor cells [13-15]. CXCL12 and CXCR4 combine to mediate the attraction of bone marrow stroma to tumor cells; and E-selectin is thought to have a critical function in allowing the breast tumor cells to enter bone marrow. The recruited endothelial cells participate in angiogenesis, which is conducive to tumor cell migration to the bone marrow ME via the circulatory system, and lymphocytes are correlated with immune regulation. The joint participation of the two factors induces transformation in the bone metastasis ME.

Rac-dependent intracellular signaling is activated through the binding of CXCL12 to CXCR4, which then initiates a cascade resulting in tumor proliferation and metastasis. A study by Liu et al. employing a model of SDF- $1 \alpha$ presented to bSDF (matrix-bound SDF-1 1 a delivered to cells at their ventral side) compared with SSDF (with soluble SDF-1 1 added to cells spread on polyelectrolyte films), showed its ability to increase cell migration and protrusion by staining actin in the cytoskeleton of breast tumor cells [16]. Specifically, the CXCL12/CXCR4 signaling pathway leads to the activation of Janus-activated kinase (JAK)/signal transducer (STAT), and other molecules, which in turn target the downstream nuclear factor $\kappa \mathrm{B}(\mathrm{NF \kappa B})$ to participate in the regulation of gene transcriptional activity [17]. This process enhances tumor proliferation by regulating the expression of related proteins. For instance, the activation of the phosphoinositide 3-kinase (PI3K)/AKT and mitogen-activated protein kinase (MAPK)/extracellular signal-regulated kinase (ERK) signaling pathways causes increased activity of downstream matrix metalloproteinases (MMP); consequently, the degradation of the collagen components presents in adjacent tissues, including the extracellular matrix and basement membrane, promotes the mobility and invasiveness of the tumor cells [18]. 
MMPs are involved in bone resorption during tumor metastasis [19], and CXCL12/CXCR4 interactions have been assumed to be important pro-malignant properties of CAFs, thus suggesting that CXCR4 suppression might be a strategy for clinical treatment of malignant tumors. AMD3100 (plerixafor), a selective antagonist of CXCR4 that is mainly used to treat non-Hodgkin lymphoma and multiple myeloma by regulating the migration of lymphocytes and hematopoietic stem cells, has been shown to have antitumor proliferation and anti-metastatic effects [20-22].

In addition to stimulating tumor cells growth through the paracrine pathway, CXCL12 secreted from CAFs also functions to regulate the maintenance and transformation of phenotypes in an autocrine manner. Kojima et al. have demonstrated the role of self-stimulating SDF-1aCXCR4 autocrine signaling in myofibroblast differentiation. Moreover, the expression of aSMA is attenuated when using short hairpin RNAs (shRNA) to inhibit the expression of CXCL12 and CXCR4 [23].

Breast cancer cells acquire traits that are similar to that of the bone marrow ME under CXCL12 and IGF1-driven selection. Breast cancer cells acquire random prometastatic mutations throughout the metastatic process by which several metastatic traits are acquired. In a landmark study, Zhang et al. reported a link between CAF numbers in a tumor with bone metastasis in triple-negative breast tumors. GSEAs (gene set enrichment analyses) showed that CAFs share several gene signatures associated with the SRS+ status of breast cancer cells, including CXCL12, CXCL14, and IGF1. Importantly, the Src response signature (SRS) score also correlates with the expression of the CAF marker aSMA, which is overexpressed in bone metastasis, thus causing a higher propensity of relapse in the bone. In contrast, MDA231-CI cells with a high level of pY416-Src have no metastasis and colonization advantages in the lung [24]. This study demonstrated that two main factors, which were identified as CXCL12 and IGF1, mediated the effects of selection in primary tumors and secondary organs in the presence of abundant CAFs [24].

By enhancing the expression of several cytokines, such as IGF1, CXCL12, and Src, this process allows metastatic breast cancer cells to orchestrate the skeletal remodeling (referred to as osteomimicry) involved in bone tropism metastasis [25, 26]. Indeed, reports have indicated that the non-receptor tyrosine kinase Src is involved in multiple processes closely related to breast cancer, including breast cancer cell migration and invasion [27], chemotherapy resistance [28], bone activity, and the EMT $[29,30]$. A previous study showed that MDA-MB-231 cell invasion and metastasis could be effectively suppressed by targeting Src, which was thought to be an upstream activator of the PI3K/Akt and Ras/Raf/ERK pathways [31]. Importantly, MMP and cathepsin S (CTSS) play key roles in the latter process through the inhibition of angiogenesis and the degradation of ECM components, and they share a common pathway with CXCL12 to some extent. Ample CXCL12 and IGF1 are expressed by CAFs, and stroma-driven selection is mediated by the accumulated factors in the tumor matrix, which act on tumor cells directly and then promote selection for breast cancer cells with higher trends to survive and colonize in the "soil" (bone marrow $\mathrm{ME}$ ). As a result, it has been postulated that "seeds" (breast cancer cells with Src hyperactivity) presenting intrinsic traits that can lead to the occurrence of bone metastases are linked to oncogenic mutations in the primary tumor [24].

\section{CAFs support and promote bone metastasis of breast cancer CSCs}

CSCs represent the true "seeds" necessary during the metastatic process. CSCs are a subpopulation of tumor cells that share the same cell markers as ordinary stem cells. Although CSCs are a rare subset residing within tumors, it has been postulated that they play a pivotal role in tumor initiation, recurrence, and metastasis via stem cell-like properties, such as self-renewal and differentiation [32]. Compared with the limited regeneration ability after differentiation of tumor cells, CSCs lying on the top of the cellular hierarchy model exhibit a high capacity for tumorigenicity and the potential to form metastases. In addition, CSCs determine tumor heterogeneity, dormancy after metastasis, and plasticity, which are all closely related to clinical tumor recurrence and resistance to radiotherapy and chemotherapy [33]. As a result, tumor metastasis is primarily driven by the migration and homing of CSCs. Lawson et al. isolated metastatic cells from PDX (patient-derived xenograft) mice with a highly sensitive, species-specific fluorescence-activated cell sorting (FACS)-based assay and then demonstrated that low-burden metastatic cells with a basal/stem cell phenotype displayed considerable tumor-initiating ability and could give rise to luminal-like tumor cells. Importantly, tumor cells, including CTCs (circulating tumor cells) and DTCs (disseminated tumor cells), which represent the main components of metastases, exhibit a more basal/stem cell-like signature. Together, these findings support the indispensable role of CSCs in metastasis, especially in the early stage [34].

CAFs interact with CSCs to form a mutually reinforcing crosstalk. Emerging evidence has shown that CSCs and CAFs can affect each other via reciprocal interactions and paracrine crosstalk. Using CD49f as a marker of CSCs in immunofluorescence assays, Valenti et al. identified the location of CSCs, which were proximal to vimentin-positive CAF-like structures in the Wnt-Met mammary gland tumors, thus supporting the existence of direct interactions between CSCs and CAFs [35]. In lung cancer, cytokines released by CAFs, such as interleukins (IL- 6 and IL-8), and TGF $\beta$, have been suggested to participate in the promotion of CSCs metastasis and stem maintenance through downstream signaling pathways and molecules, such as TGF $\beta /$ Smad and STAT3, which eventually leads to tumor chemotherapy resistance $[35,36]$. In addition, Jung et al. found that overexpression of 
CXCL12 $\gamma$ led to a significant increase in the expression of the CSC phenotype and facilitated the development of spheres in culture [37]. Therefore, the self-renewal of CSCs is likely also regulated by the CXCL12/CXCR4 axis. The role of the CXCL12/CXCR4 axis in maintaining the stemness of breast tumor cells is also supported by the studies linking it to IL-7 expressed by CAFs [38].

By providing a niche to harbor CSCs and upregulating the expression of factors influencing the surrounding immune cells and CSCs, CAFs function as helpers to assist and support the metastatic process of mammary carcinoma [38]. Bone marrow and CAFs can modify the pre-metastatic ME and act on CSCs through cytokines, such as IL- $1 \beta$ and TGF $\beta$, and play an important role in breast cancer cell colonization of bone [39]. Among the many pathways, the Hedgehog $(\mathrm{Hh})$ signaling pathway has been studied more in-depth because of its complexity and comprehensiveness in reciprocal interactions between CSCs and CAFs. For example, based on the murine M6-Hh tumor allograft model (a low-grade triple-negative breast cancer (TNBC) driven by transgenic Hh expression). Cazet et al. demonstrated that $\mathrm{Hh}$ signaling is involved in promoting both CAF activation and CSC plasticity. Using an RNA-Seq approach, Hh signaling was found to function as an activator of stromal gene signatures (HSGS), including Col4a1, Tspan11, St8sia2, and Tnfaip6, which are related to ECM in CAFs; moreover, this process is linked to stromal remodeling, which subsequently promotes cancer stemness and can be inhibited by smoothed inhibitors (SMOi) [40].

The Hh pathway can also activate the fibroblast growth factor (FGF5) signaling in CAFs to mediate their acquisition of a CSC phenotype, which is associated with chemotherapy resistance [40]. Moreover, the hedgehog ligand sonic hedgehog $(\mathrm{SHH})$ secreted from CSCs in a paracrine pathway activates CAFs by upregulating the expression of aSMA and fibronectin and by increasing the secretion of activin, IGF1, and leukemia inhibitory factor (LIF) [35]. As mentioned previously, CAFs are extremely heterogeneous and have different sources. With their multi-directional differentiation ability, CSCs are hypothesized to be one of the key sources of CAFs in addition to fibroblasts and MSCs. Another study by Nair et al. provided the first evidence that CSCs can differentiate into CAF-like cells in the tumor niche. Myofibroblasts separated from colonization were composed of CSCs generated from mouse induced pluripotent stem (miPS) cells. As proven by their sphere-forming capacity, these cells showed a CAF-like phenotype with high expression of aSMA, fibroblast-specific protein-1 (FSP1), vimentin, CXCL12, and TGF $\beta$ surrounding the spheres [41].

CD44 is required for bone metastasis of breast cancer CSCs. In general, a cluster of differentiation 44 (CD44) is likely the most widely used CSC marker, and it also plays an important role in CSCs self-renewal, communication with the ME, and maintenance of stemness, which have been linked to tumorigenesis, metastasis, and therapeutic resistance [32]. CD44 is a class I transmembrane glycoprotein that can be used as a surface receptor adhesion molecule that binds to extracellular matrix proteins, including HA, OPN, laminin, collagen, and fibronectin. As a principal component of the ECM, HA is the main ligand of CD44, and ECM mechanics, such as the HA-CD44 axis, also contribute to the cell-cell and cell-matrix adhesion, migration, proliferation, and differentiation involved in tumor progression [42, 43].

HA/CD44 axis promotes EMT and anoikis resistance that is necessary for metastasis. HA combined with CD44 can induce the occurrence of EMT via epidermal growth factor (EGF) signaling activated by TGF $\beta 1$ [44]. Using 4-methylumbelliferine (4-MU) to inhibit hyaluronan synthase (HAS), which regulates the synthesis and degradation of hyaluronan and shRNA and subsequently disrupts the expression of CD44, the EMT process was found to slow down along with a decreased expression of the EMT associated proteins including N-cadherin, Snail, Twist, and Zeb1 [44].

A large number of studies have demonstrated that EMT acts as a pivotal event in tumor progression, especially tumor cell metastasis. By undergoing the EMT, in which the adhesion molecules are downregulated, breast cancer cells, including CSCs, acquire increased plasticity and migration ability. Additionally, the EMT protects tumor cells from anoikis, which leads to the failure of single-cell metastasis. Moreover, the HA/CD44 axis is crucial to anoikis resistance in an EMT-independent manner. Cieply et al. reported that CD44S could rescue human mammary epithelial (HMLE)Twist-ER cells that had undergone the EMT with low expression of CD44 from anoikis and that it failed in the HA non-binding mutant. Conversely, the EMT also upregulated $\mathrm{HA}$ and CD44S and thus enhanced CD44-cytoskeletal interactions to participate in cell migration [45]. This result proves the mutual effect between the HA/CD44 axis and EMT. After reaching the distant target sites through the circulation and establishing secondary metastatic colonization, those tumor cells reversed the phenotype via the MET in which epithelial markers were re-acquired [46].

The occurrence of the MET in metastases is closely related to CAFs [46]. In the mouse breast cancer MMTV-PyMT model, Del Martin et al. demonstrated that MICs (metastatic initiating cells) that possess partial mesenchymal features linked to tumor metastatic competence are capable of activating fibroblasts and that those activated CAFs could, in turn, mediate the upregulation of epithelial markers in MICs via TGF $\beta$ signaling when the metastatic tumor cells start to grow [47].

Role of the OPN/CD44 axis in osteolytic metastasis of breast cancer. OPN, also called secreted phosphoprotein 1 (SPP1), is another ligand that binds to CD44 to regulate cellmatrix adhesion and signaling in the primary and secondary tumor ME. OPN is considered to be a glycoprotein linked to bone disorders. Thus, it has been postulated that OPN plays a central role in bone ME during tumor metastasis by constructing interactions with different cells, such as macrophages, tumor cells, CAFs, osteoclasts, and osteoblasts 
[48]. For example, Rao and colleagues found that macrophages infiltrating colorectal cancer can be activated by OPN secreted from tumor cells, which in turn promoted tumorigenicity and clonogenicity by the educated macrophages via the JUN N-Terminal Kinase (JNK) pathway, which is activated by the OPN/CD44 axis [49].

Using a MC3T3-E1 cell model, another study by Kusuyama et al. found that OPN exerts negative effects on osteoblasts related to mechanical stimulation, which can be partly explained by nitric oxide (NO) production, and by attenuating focal adhesion kinase (FAK) signaling pathways through the induction of low molecular-weight protein tyrosine phosphatase (LMW-PTP), which requires a combination of OPN with CD44; these results suggest a negative role of the OPN/CD44 axis in osteoblast responses to mechanical stress [50]. Simultaneously, both HA and OPN, as ligands combined with CD44 in the matrix, have been shown to promote osteoclast differentiation and function by upregulating nuclear factor $\mathrm{\kappa B}$ ligand (RANKL) expression [51]. Immunohistochemical examinations have shown the colocalization of CD44, OPN, and HA in bone ME, which revealed that cell-matrix interactions involved with CD44 are essential for bone metastasis in forms of osteolysis [51].

Direct interactions between OPN, HA, CD44, and CAFs. CD44 is overexpressed on CAFs in some conditions. Kinugasa et al. demonstrated a strong correlation between CD44 expression on the surface of CAFs and hypoxia and hypo nutrition [52]. Simultaneously, CD44 expressed on CAFs is involved in maintaining tumor stemness and drug resistance [52]. Although the exact mechanism remains incompletely understood, it could be partly explained by the HA/CD44 and the OPN/CD44 axes, which could mediate the effects of CD44 on CSCs. Indeed, CD44 is also involved in the CXCR4mediated cell response induced by CXCL12. A study by Liu et al. demonstrated that the HA adhesion receptor CD44 has a more than complementary and possibly even an indispensable role in promoting MDA-MB-231 cancer cell spread, migration, and protrusion formation by changing the phenotype of the cytoskeleton, including lamellipodia and filopodia, and ERK signaling in the CXCL12-CXCR4 axis [23]. Additionally, according to a study by Kuo et al., the migration of MSCs is also characterized as OPN-dependent, and they followed the migration of tumor cells to the bone marrow [53]. MSCs have been shown to transition to CAFs in the tumor microenvironment (TME). Interestingly, further analysis revealed that the transition of MSCs to CAFs is OPN-dependent and that it occurs prior to the increase of tumor stemness in TME. As mentioned before, CAFs function to support CSCs and maintain the stemness of tumors.

The bone ME is extensively enriched with the CD44 ligands OPN and HA. The overexpression of CD44 in CSCs is an important factor in the involvement of CSCs in breast cancer metastasis. In summary, these findings suggest that cancer stemness is regulated by the tumor ME and that CAFs participate in creating the bone marrow premetastatic niche and increasing cancer stemness (Figure 2).

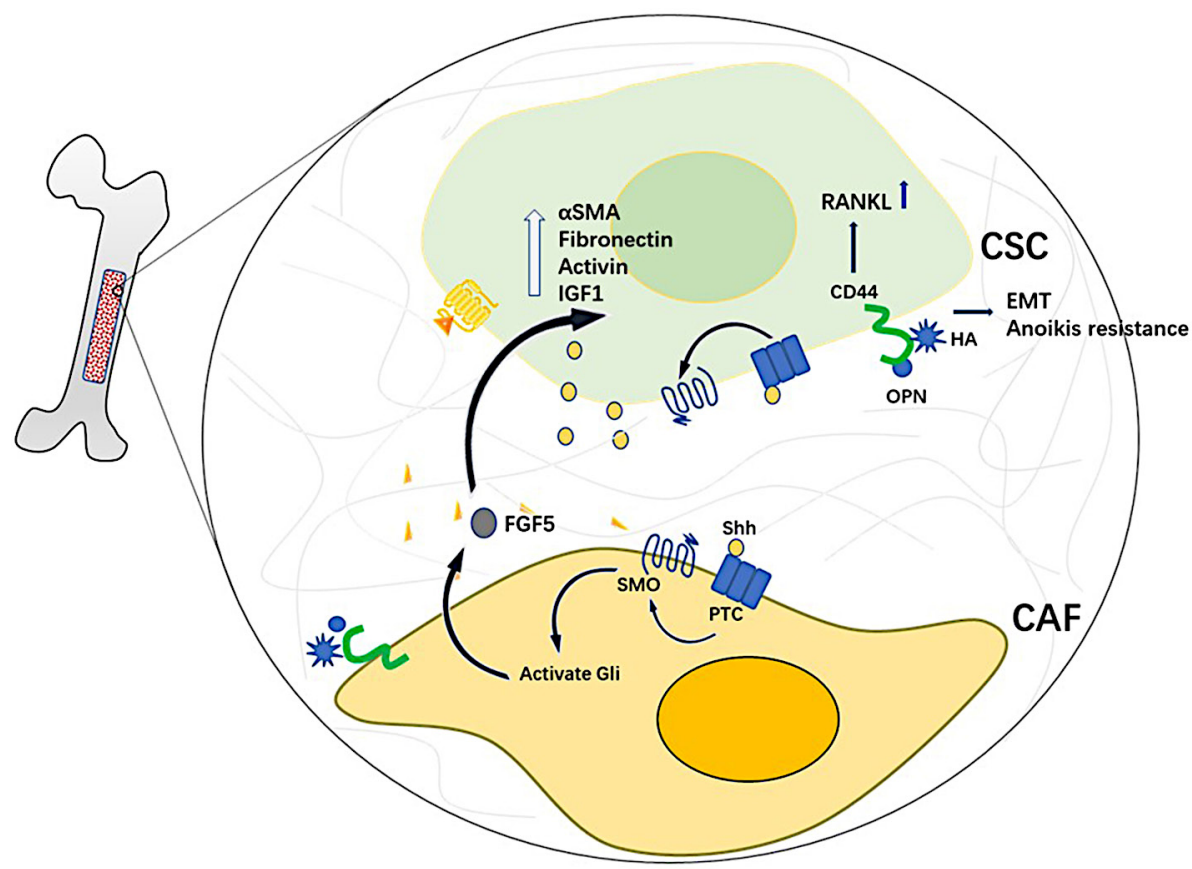

Figure 2. Reinforcing crosstalk between CSC and CAF in the bone microenvironment. In this process, the following pathways are involved: hedgehog signaling (ligands from CSC not only participate in the continuous activation of CAF but also affect their own characteristics through autocrine or secondary products from CAF), OPN/CD44 and HA/CD44 axis (this pathway can promote the occurrence of EMT and anoikis resistance, which imparts strong vitality to CSCs that are highly expressed by CD44 in bone microenvironments, and it can also modify the bone microenvironment by affecting osteogenesis and osteolysis). 


\section{CAFs-derived exosomes regulate the pre- metastatic ME as a pre-requisite for the occurrence of bone metastases in breast cancer}

Formation and properties of PMNs. PMNs are a selected tissue site for tumor cells that are formed by the effects of cytokines secreted from tumor cells or tumor stroma before metastasis. PMNs are a "suitable soil" for "seeds" to engraft and grow and are related to further cancer progression. Specifically, PMNs are characterized by altered metabolism pathways, ECM, and immune suppression and provide a supportive ME for outgrowth, survival, adherence, and invasion of tumor cells. Several factors are involved in promoting PMNs formation, including miRNAs. Simultaneously, exosomes delivering miRNAs play a central role in establishing PMNs under the influence of the tumor cells and stroma.

In addition to communicating via direct cell-cell contact, tumor cells and CAFs release cytokines or exosomes to mediate paracrine cellular interactions during tumor progression. Therefore, CAFs affect the organ selectivity of tumor metastasis by secreting exosomes, which has been verified in several experiments (Table 1). Exosomes are nano-sized vesicles (40-100 $\mathrm{nm}$ in diameter) derived from various cell types in the form of membrane budding that can establish a connection between tumor cells, tumor stroma, and distant organs by delivering cytokines, active growth factors, and functional DNA fragments as well as non-coding and coding RNAs [54-56].

Simultaneously, studies have proposed that these exosomes are capable of regulating the selective tropisms of cancer cells for target organs through the use of specific exosomal integrins fused with the ECM of distant organs after entering the circulation system. Exosomes function to educate cells to alter their phenotype or metabolism pathways in the target tissues by releasing their contents before the primary tumor cells metastasize. Different integrins result in different organ affinities of exosomes, thus explaining the different organotropisms of secondary metastasis in various types of cancer [57]. For example, B02 cells (MDA-MB- 231 breast cancer cells characterized by the overexpression of $\operatorname{av} \beta 3$ integrin) only metastasize to bone, and this finding was supported by other studies and analyses in humans and animals [58]. PSK1404mediated targeting of integrin $\alpha v \beta 3$ inhibits bone metastasis and reduces bone destruction. Moreover, exosomal integrins $\alpha 6 \beta 4$ and $\alpha 6 \beta 1$ are related to lung metastasis and integrin av $\beta 5$ is associated with liver metastasis [57].

miRNAs transported by exosomes cause imbalances in bone formation and absorption. miRNAs are a class of small non-coding RNAs transcribed by RNA polymerase, and they combine with the "seed sequence" of the target mRNAs through RISC (RNA induced silencing complex). miRNAs play important roles in multiple physiological and pathological cellular processes, including differentiation, proliferation, apoptosis, and tumorigenesis. Homeostasis of bone microenvironment, mainly kept by osteoclasts and osteoblasts, can be disrupted and thus transformed from normal niche to metastatic niche. This complicated progression can generally be explained by the "vicious cycle of bone metastasis", positive feedback of the process of osteolysis and tumor growth initiated by the tumor cells once they have established a foothold. TGF $\beta$ derived from bone resorption activates the process through the upregulation of PTHrP, Jagged1, and RANKL. Meanwhile, multiple growthpromoting factors are generated in this process [66]. Thus, a prerequisite for the generation of osteolytic metastases is the relative hyperfunction of osteoclasts. In this process, miRNA serves three primary functions by influencing the

Table 1. Summary of CAF-derived exosomes and mechanisms involved in cancer progression.

\begin{tabular}{|c|c|c|c|c|}
\hline Cancer & Main exosomal contents & Recipient cells & Role/Mechanism & Reference(s) \\
\hline CRC & miR-21 & CRC & Promote liver metastasis through upregulating miR-21 in CRC & [59] \\
\hline $\mathrm{BC}$ & miR-181d-5p & $\mathrm{BC}$ & $\begin{array}{l}\text { Accelerate EMT and promote proliferation by regulating CDX2/ } \\
\text { HOXA5 }\end{array}$ & {$[60]$} \\
\hline SACC & TSP1, LOXL2, and MMP2 & LF & $\begin{array}{l}\text { Promote pre-metastatic niche formation in the lungs via enhanc- } \\
\text { ing ECM remodeling }\end{array}$ & [61] \\
\hline OSCC & $\operatorname{miR}-34 a-5 p$ & OSCC & $\begin{array}{l}\text { Facilitate proliferation and metastasis of OSCC by targeting AXL } \\
\text { and downstream signaling cascade }\end{array}$ & [62] \\
\hline $\mathrm{HNC}$ & miR-196a & HNC & $\begin{array}{l}\text { Promote HNC cell proliferation and survival by targeting CD- } \\
\text { KN1B and ING5 }\end{array}$ & [63] \\
\hline CRC & lncRNA H19 & $\mathrm{CRC}$ & $\begin{array}{l}\text { Enhance stemness and chemoresistant by activating the } \\
\beta \text {-catenin pathway }\end{array}$ & [39] \\
\hline PDAC & miR-146a and snail & PDAC & $\begin{array}{l}\text { Account for gemcitabine resistant with upregulated Snail and } \\
\text { miR-146a carried by exosomes }\end{array}$ & [64] \\
\hline PC, PDAC & $\begin{array}{l}\text { TCA cycle metabolites, amino } \\
\text { acids, and lipids }\end{array}$ & PC, PDAC & $\begin{array}{l}\text { Lead to metabolism reprogramming via the delivery of metabo- } \\
\text { lites to fuel recipient cells }\end{array}$ & [65] \\
\hline
\end{tabular}

Abbreviations: CRC - colorectal cancer; BC - breast cancer; CDX2 - caudal-related homeobox 2; HOXA5 - homeobox A5; SACC - salivary adenoid cystic carcinoma; TSP1 - thrombospondin-1; LOXL2 - lysyl oxidase 2; MMP2 - metalloproteinases 2; LF - lung fibroblast; OSCC - oral squamous cell carcinoma; HNC - head and neck cancer; PDAC - pancreatic ductal adenocarcinoma; PC - prostate cancer; lncRNA H19 - long noncoding RNA H19; TCA - tricarboxylic acid 
Table 2. Selected miRNAs important for bone metastasis of breast cancer.

\begin{tabular}{|c|c|c|c|}
\hline miRNA & Function(s) & Target gene(s) & Reference(s) \\
\hline $\operatorname{miR}-20 a-5 p$ & $\begin{array}{l}\text { Shows high expression in TNBC } \\
\text { Promotes invasion and metastasis of TNBC cells } \\
\text { Accelerates the differentiation and proliferation of osteoclasts }\end{array}$ & SRCIN1 & {$[72]$} \\
\hline $\operatorname{miR}-214-3 p$ & $\begin{array}{l}\text { Upregulated during osteoclastogenesis } \\
\text { Stimulated by Enoxacin to inhibit osteoclast formation and bone metastasis } \\
\text { Transferred from osteoclasts to osteoblasts, inhibiting bone formation }\end{array}$ & TRAF3 & {$[81-83]$} \\
\hline $\operatorname{miR}-218$ & $\begin{array}{l}\text { Highly detected during bone metastasis of breast cancer } \\
\text { Decreases type I collagen secretion to inhibit collagen deposition by osteoblasts and osteo- } \\
\text { blast differentiation } \\
\text { Increases inhibin } \beta \mathrm{A} \text { and consequently activates SMAD signaling and upregulates Timp3 } \\
\text { expression }\end{array}$ & $\begin{array}{l}\text { COL1A1 } \\
\text { YY1 } \\
\text { INHBB }\end{array}$ & {$[84]$} \\
\hline miR-30 & Suppresses tumors in breast cancer & $\begin{array}{l}\text { CDH11 } \\
\text { ITGA5 } \\
\text { ITGB3 }\end{array}$ & {$[69]$} \\
\hline $\operatorname{miR}-182$ & Promotes osteoclastogenesis and bone metastasis by regulating TGF $\beta$ signaling & SMAD7 & {$[85]$} \\
\hline $\operatorname{miR}-106 b$ & Decreased during bone metastasis of breast cancer & MMP2 & {$[86]$} \\
\hline $\operatorname{miR}-181 b$ & $\begin{array}{l}\text { Downregulated during Raw } 264.7 \text { cells differentiated into osteoclasts } \\
\text { Influences osteoclastogenic factors through miR-181b/OSM axis }\end{array}$ & OSM & {$[87]$} \\
\hline
\end{tabular}

transcription process of factors involved in proliferation, vascularization, and immunosuppression [67, 68]. Breast cancer cell-derived miRNAs are transported to the bone ME via exosomes, dysregulate the differentiation of osteoblasts and osteoclasts and disrupt the balance of the bone remodeling system, which leads to abnormal bone resorption and destruction, and promote the formation of suitable PMNs for breast cancer cells. For instance, miR-218 inhibits the formation and deposition of type 1 collagen by osteoblasts through downregulating COL1A1 expression and upregulating inhibin $\beta A$ expression, and the latter function is based on the induction of Timp3, which is the inhibitor of the N-procollagenase ADAMTS2. Simultaneously, inhibin $\beta$ A activates SMAD signaling in MDA-23 cancer cells to facilitate tumor proliferation [69].

Several studies have identified the functions of miRNAs in driving cell differentiation and phenotype modulation to promote bone metastasis by affecting the internal balance, which is likely shifted towards osteolysis bone lesions (Table 2). Similarly, various factors, cytokines, and hormones are involved in regulating osteogenesis, and among these factors, macrophage colony-stimulating factor (M-CSF) and receptor activator of RANKL are considered the most indispensable pathways [70]. For example, during osteoclastogenesis [71-73], the RANKL-induced expression of miR-21 downregulates the levels of programmed cell death 4 (PDCD4), a negative regulator of osteoclastogenesis, and it is defined as an oncogenic microRNA along with miR-10b, miR155, and miR-125a [71].

Research by Hashimoto and colleagues found that hsa-miR940 transferred by exosomes can mediate MSC differentiation into osteoblasts and induce osteoblastic lesions, even in the bone metastasis ME of MDA-MB-231 cells, which is often described as causing osteolytic destruction [72]. In summary, the imbalance of osteoblasts and osteoclasts caused by cancer-related miRNAs subsequently leads to the dysregulation of cytokines and factors in the bone $\mathrm{ME}$, thus affecting various signaling pathways to potentiate the formation of bone metastases.

Exosomes induce the EMT and tumor-related blood vessel formation. Emerging evidence has shown that exosomal transmission plays roles in EMT/MET, angiogenesis, extracellular matrix remodeling, immunosuppression, and metabolic changes. As previously mentioned, the EMT is a biological process that enables tumor cells to develop superior motility while metastasizing. Using models of MDA-MB-231 and 4T1 cells exposed to exosomes isolated from p $85 a-/-$ fibroblasts, Chen et al. found that the EMT is induced via the canonical Wnt/ $\beta$-catenin signaling pathway, which is activated by the Wnt $10 \mathrm{~b}$ that is abundant in p85a-/fibroblasts and released from CAF-derived exosomes [2]. Moreover, exosomes also maintain the EMT phenotype of breast cancer cells and endow them with anoikis-resistance traits [74]. However, when exosomes are removed, the reverse process, i.e., MET, occurs and restores the epithelial phenotypes [74]. As such, this process is believed to be regulated by the factors released from tumor cells through mechanisms other than exosomes in the TME [53]. However, the regulation of exosomes and the mechanism by which tumor cells regain an epithelial phenotype via the EMT are still unclear. The former can be partly explained based on the study by Lin et al., who proposed that ASPH, which is detectable in $90.1 \%$ of breast cancer patients, is responsible for guiding the synthesis, release, and delivery of metalloproteinaseenriched exosomes by activating the Notch cascade in breast cancer [75].

Exosomal Annexin A2 (Exo-AnxA2) promotes tPA-dependent angiogenesis in PMNs during the breast cancer metastasis process [76]. AnxA2-p11-tPA-mediated plasmin generation acts to promote endothelial cell migra- 
tion, which is essential for angiogenesis [77]. The effects of Exo-AnxA2 on the migration and invasion of endothelial cells were demonstrated by Maji et al. in a human umbilical vein endothelial cell (HUVEC) model, which showed that migration and invasion were significantly inhibited by LCKLSL, an AnxA2 competitive inhibitory peptide [76]. In addition to inducing tumor-associated blood vessel formation, the transfer of Annexin A2 by exosomes also leads to the activation of macrophages and the upregulation of IL- 6 and tumor necrosis factor (TNF- $\alpha$ ), thereby participating in PMN formation [76].

Exosomes/micro-vesicles mediate immunosuppression and metabolic reprogramming in PMNs. Tumorassociated macrophages (TAMs) resemble M2 macrophages marked with CD163 and CD106, represent a considerable part of the tumor stroma, and play roles in many stages of cancer progression [78]. TAMs exhibit strong immunosuppressive abilities by expressing programmed death-ligand 1 (PD-L1) and disrupting the functions of natural killer (NK) and T cells [78]. A study by Yavuz et al. showed that the differentiation of M1 macrophages into TAMs can be redirected by CAFs, especially in breast cancer, and a high number of CAFs is correlated with the abundance of TAMs, which impact the immunosuppression and acquisition of the EMT phenotype [15]. Interestingly, research by Bissau et al. showed that exosomes released from breast tumor-educated MSCs promote monocytic myeloid-derived suppressor cells (M-MDSCs) to differentiate into M2 polarized macrophages, which resemble TAMs and express high levels of immunosuppressive factors, including IL-10, L-arginase, CD206, and PD-L1 [79]. The contents of those exosomes were investigated by tandem mass spectrometry, and they included TGF- $\beta$, C1q, and semaphorin [79].
In contrast, the differentiation of dendritic cells from bone marrow precursors could be hindered by the presence of breast tumor exosomes. Mechanistically, IL-6 induced by TS/A exosomes inhibits the differentiation of myeloid progenitor cells into dendritic cells by activating the JAK2/ STAT3 pathway, which has been shown to be important in tumor growth and metastasis $[70,80]$. In summary, suppressive factors related to the exosomes from tumor stroma, including CAFs and TAMs, downregulate the function of the immune system to produce immunosuppressive PMNs.

By transferring miRNA, cancer secreted extracellular vehicles (EVs) also contribute to the generation of extensive intercellular crosstalk, which enables metabolic reprogramming in the breast tumor stroma [73]. An MYC-miR105-MYC activation loop plays a pivotal role in extensively mediating the effects of MDA-MB-231 breast cancer cellderived EVs on the TME and PMNs [71]. By redirecting the metabolic mechanisms and detoxifying several metabolic by-products, including lactic acid and ammonium, miR-105reprogrammed CAFs enhance the flexibility of tumor energy utilization and are involved in favorable PMN formation via different mechanisms according to the local milieu [71]. For example, when nutritional substrates are sufficient, miR-105reprogrammed CAFs promote glucose and glutamate metabolism to fuel surrounding tumor cells, and under these nutritional conditions, miR-105-reprogrammed CAFs inhibit the tricarboxylic acid cycle [71].

In conclusion, distant organ metastasis is a complicated event related to distinctive factors associated with the significant alterations of breast tumor cells and the extensive crosstalk that remodels the ME, which are both associated with breast cancer metastasis. As emphasized here, the skeletal system contains many notable cytokines

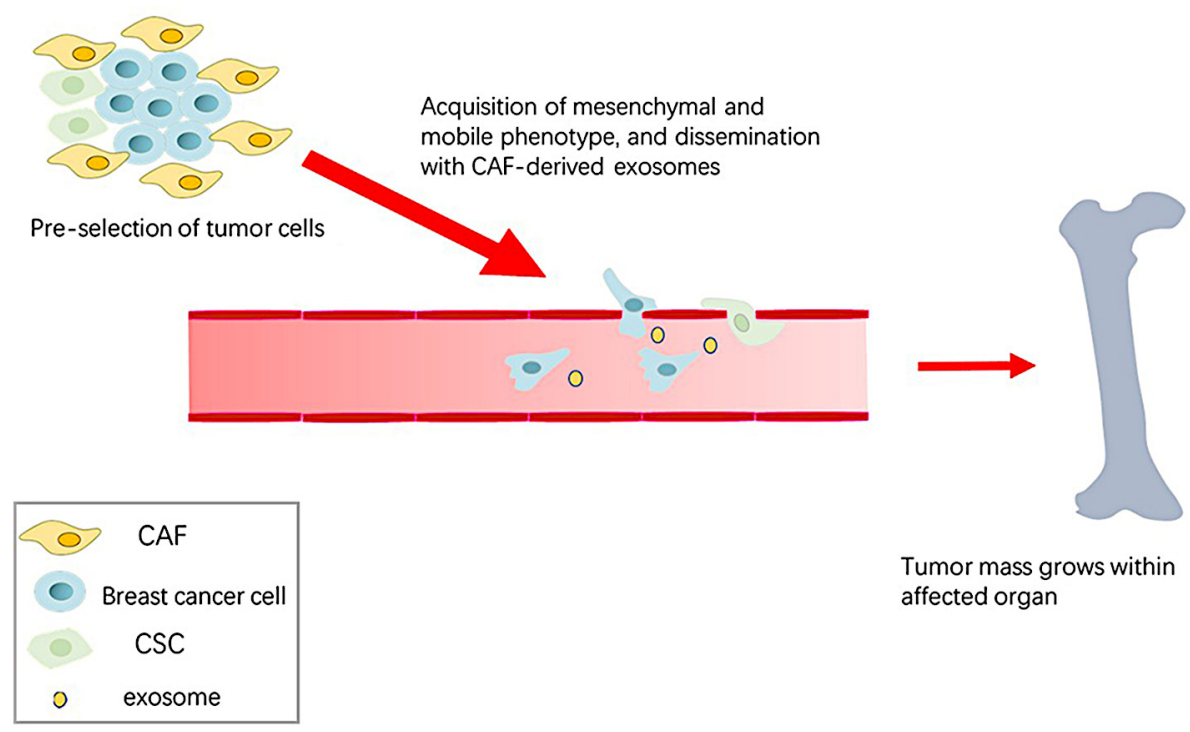

Figure 3. Process of breast cancer bone metastasis. CAF is extensively involved in this process from tumor cell pre-selection to PMN formation. 
and molecules. Several of these cytokines are related to CAFs, which are key components in the tumor stroma that exert significant supportive functions on tumor progression throughout the whole metastatic process from "seed" selection to distant organ colonization (Figure 3). Current studies have indicated the importance of CAFs, and with the development of single-cell analysis methods, more specific and crucial mechanisms are expected to be demonstrated. Thus, targeted drugs that treat bone metastasis of breast cancer could be developed.

\section{References}

[1] KARNOUB AE, DASH AB, VO AP, SULLIVAN A, BROOKS MW et al. Mesenchymal stem cells within tumour stroma promote breast cancer metastasis. Nature 2007; 449: 557-563. https://doi.org/10.1038/nature06188

[2] CHEN Y, ZENG C, ZHAN Y, WANG H, JIANG X et al. Aberrant low expression of p85alpha in stromal fibroblasts promotes breast cancer cell metastasis through exosome-mediated paracrine Wnt10b000. Oncogene 2017; 36: 4692-4705. https://doi.org/10.1038/onc.2017.100

[3] MCCARTHY JB, EL-ASHRY D, TURLEY EA. Hyaluronan, Cancer-Associated Fibroblasts and the Tumor Microenvironment in Malignant Progression. Front Cell Dev Biol 2018; 6: 48. https://doi.org/10.3389/fcell.2018.00048

[4] CHEN X, SONG E. Turning foes to friends: targeting cancer-associated fibroblasts. Nat Rev Drug Discov 2019; 18: 99-115. https://doi.org/10.1038/s41573-018-0004-1

[5] FIORI ME, DI FRANCO S, VILLANOVA L, BIANCA P, STASSI G et al. Cancer-associated fibroblasts as abettors of tumor progression at the crossroads of EMT and therapy resistance. Mol Cancer 2019; 18: 70. https://doi.org/10.1186/ s12943-019-0994-2

[6] KWA MQ, HERUM KM, BRAKEBUSCH C. Cancer-associated fibroblasts: how do they contribute to metastasis? Clin Exp Metastasis 2019; 36: 71-86. https://doi.org/10.1007/ s10585-019-09959-0

[7] JOTZU C, ALT E, WELTE G, LI J, HENNESSY BT et al. Adipose tissue-derived stem cells differentiate into carcinoma-associated fibroblast-like cells under the influence of tumor-derived factors. Anal Cell Pathol (Amst) 2010; 33: 61-79. https://doi.org/10.3233/ACP-CLO-2010-0535

[8] RAZ Y, COHEN N, SHANI O, BELL RE, NOVITSKIY $\mathrm{SV}$ et al. Bone marrow-derived fibroblasts are a functionally distinct stromal cell population in breast cancer. J Exp Med 2018; 215: 3075-3093. https://doi.org/10.1084/ jem. 20180818

[9] BADO I, GUGALA Z, FUQUA SAW, ZHANG XH. Estrogen receptors in breast and bone: from virtue of remodeling to vileness of metastasis. Oncogene 2017; 36: 4527-4537. https://doi.org/10.1038/onc.2017.94

[10] GAO Y, BADO I, WANG H, ZHANG W, ROSEN JM et al. Metastasis Organotropism: Redefining the Congenial Soil. Dev Cell 2019; 49: 375-391. https://doi.org/10.1016/j.devcel.2019.04.012
[11] TENG F, TIAN WY, WANG YM, ZHANG YF, GUO F et al. Cancer-associated fibroblasts promote the progression of endometrial cancer via the SDF-1/CXCR4 axis. J Hematol Oncol 2016; 9: 8. https://doi.org/10.1186/s13045-015-0231-4

[12] DOMANSKA UM, KRUIZINGA RC, NAGENGAST WB, TIMMER-BOSSCHA H, HULS G et al. A review on CXCR4/ CXCL12 axis in oncology: no place to hide. Eur J Cancer 2013; 49: 219-230. https://doi.org/10.1016/j.ejca.2012.05.005

[13] KALLURI R. The biology and function of fibroblasts in cancer. Nat Rev Cancer 2016; 16: 582-598. https://doi. org/10.1038/nrc.2016.73

[14] OUYANG L, CHANG W, FANG B, QIN J, QU X et al. Estrogen-induced SDF-1a production promotes the progression of ER-negative breast cancer via the accumulation of MDSCs in the tumor microenvironment. Sci Rep 2016; 6: 39541. https://doi.org/10.1038/srep39541

[15] GOK YAVUZ B, GUNAYDIN G, GEDIK ME, KOSEMEHMETOGLU K, KARAKOC D et al. Cancer associated fibroblasts sculpt tumour microenvironment by recruiting monocytes and inducing immunosuppressive PD-1(+) TAMs. Sci Rep 2019; 9: 3172-3172. https://doi.org/10.1038/ s41598-019-39553-z

[16] LIU XQ, FOUREL L, DALONNEAU F, SADIR R, LEAL $S$ et al. Biomaterial-enabled delivery of SDF-1alpha at the ventral side of breast cancer cells reveals a crosstalk between cell receptors to promote the invasive phenotype. Biomaterials 2017; 127: 61-74. https://doi.org/10.1016/j.biomaterials.2017.02.035

[17] WOBUS M, LIST C, DITTRICH T, DHAWAN A, DURYAGINA $\mathrm{R}$ et al. Breast carcinoma cells modulate the chemoattractive activity of human bone marrow-derived mesenchymal stromal cells by interfering with CXCL12. Int J Cancer 2015; 136: 44-54. https://doi.org/10.1002/ijc.28960

[18] MASUDA T, ENDO M, YAMAMOTO Y, ODAGIRI H, KADOMATSU T et al. ANGPTL2 increases bone metastasis of breast cancer cells through enhancing CXCR4 signaling. Sci Rep 2015; 5: 9170. https://doi.org/10.1038/srep09170

[19] O'KEEFE RJ, GUISE TA. Molecular mechanisms of bone metastasis and therapeutic implications. Clin Orthop Relat Res 2003; 415: S100-104. https://doi.org/10.1097/01. blo.0000093847.72468.2f

[20] MURALIDHARAN R, PANNEERSELVAM J, CHEN A, ZHAO YD, MUNSHI A et al. HuR-targeted nanotherapy in combination with AMD3100 suppresses CXCR4 expression, cell growth, migration and invasion in lung cancer. Cancer Gene Ther 2015; 22: 581-590. https://doi.org/10.1038/ cgt.2015.55

[21] AZAB AK, RUNNELS JM, PITSILLIDES C, MOREAU AS, AZAB F et al. CXCR4 inhibitor AMD3100 disrupts the interaction of multiple myeloma cells with the bone marrow microenvironment and enhances their sensitivity to therapy. Blood 2009; 113: 4341-4351. https://doi.org/10.1182/ blood-2008-10-186668

[22] KANG Y, CHEN BJ, DEOLIVEIRA D, MITO J, CHAO NJ. Selective enhancement of donor hematopoietic cell engraftment by the CXCR4 antagonist AMD3100 in a mouse transplantation model. PLoS One 2010; 5: e11316. https://doi. org/10.1371/journal.pone.0011316 
[23] KOJIMA Y, ACAR A, EATON EN, MELLODY KT, SCHEEL $\mathrm{C}$ et al. Autocrine TGF-beta and stromal cell-derived factor-1 (SDF-1) signaling drives the evolution of tumor-promoting mammary stromal myofibroblasts. Proc Natl Acad Sci U S A 2010; 107: 20009-20014. https://doi.org/10.1073/ pnas. 1013805107

[24] ZHANG XH, JIN X, MALLADI S, ZOU Y, WEN YH et al. Selection of bone metastasis seeds by mesenchymal signals in the primary tumor stroma. Cell 2013; 154: 1060-1073. https://doi.org/10.1016/j.cell.2013.07.036

[25] CHU GCY, CHUNG LWK. RANK-mediated signaling network and cancer metastasis. Cancer Metastasis Rev 2014; 33: 497-509. https://doi.org/10.1007/s10555-013-9488-7

[26] KRUGER TE, MILLER AH, GODWIN AK, WANG J. Bone sialoprotein and osteopontin in bone metastasis of osteotropic cancers. Crit Rev Oncol Hematol 2014; 89: 330-341. https://doi.org/10.1016/j.critrevonc.2013.08.013

[27] POHORELIC B, SINGH R, PARKIN S, KORO K, YANG $\mathrm{AD}$ et al. Role of $\mathrm{Src}$ in breast cancer cell migration and invasion in a breast cell/bone-derived cell microenvironment. Breast Cancer Res Treat 2012; 133: 201-214. https://doi. org/10.1007/s10549-011-1753-2

[28] CAMPBELL PS, MAVINGIRE N, KHAN S, ROWLAND LK, WOOTEN JV et al. AhR ligand aminoflavone suppresses alpha6-integrin-Src-Akt signaling to attenuate tamoxifen resistance in breast cancer cells. J Cell Physiol 2018; 234: 108-121. https://doi.org/10.1002/jcp.27013

[29] TANG ZN, ZHANG F, TANG P, QI XW, JIANG J. RANKLinduced migration of MDA-MB-231 human breast cancer cells via Src and MAPK activation. Oncol Rep 2011; 26: 1243-1250. https://doi.org/10.3892/or.2011.1368

[30] OLEA-FLORES M, ZUNIGA-EULOGIO M, TACUBASAAVEDRA A, BUENO-SALGADO M, SANCHEZ-CARVAJAL A et al. Leptin Promotes Expression of EMT-Related Transcription Factors and Invasion in a Src and FAK-Dependent Pathway in MCF10A Mammary Epithelial Cells. Cells 2019; 8: 1133. https://doi.org/10.3390/cells8101133

[31] GAUTAM J, BANSKOTA S, LEE H, LEE YJ, JEON YH et al. Down-regulation of cathepsin $S$ and matrix metalloproteinase-9 via Src, a non-receptor tyrosine kinase, suppresses triple-negative breast cancer growth and metastasis. Exp Mol Med 2018; 50: 118. https://doi.org/10.1038/s12276-0180135-9

[32] YAN Y, ZUO X, WEI D. Concise Review: Emerging Role of CD44 in Cancer Stem Cells: A Promising Biomarker and Therapeutic Target. Stem Cells Transl Med 2015; 4: 1033 1043. https://doi.org/10.5966/sctm.2015-0048

[33] SRIDHARAN S, HOWARD CM, TILLEY AMC, SUBRAMANIYAN B, TIWARI AK et al. Novel and Alternative Targets Against Breast Cancer Stemness to Combat Chemoresistance. Front Oncol 2019; 9: 1003. https://doi.org/10.3389/ fonc. 2019.01003

[34] LAWSON DA, BHAKTA NR, KESSENBROCK K, PRUMMEL KD, YU Y et al. Single-cell analysis reveals a stem-cell program in human metastatic breast cancer cells. Nature 2015; 526: 131-135. https://doi.org/10.1038/nature15260
[35] VALENTI G, QUINN HM, HEYNEN G, LAN L, HOLLAND JD et al. Cancer Stem Cells Regulate Cancer-Associated Fibroblasts via Activation of Hedgehog Signaling in Mammary Gland Tumors. Cancer Res 2017; 77: 2134-2147. https://doi.org/10.1158/0008-5472.CAN-15-3490

[36] ALGUACIL-NUNEZ C, FERRER-ORTIZ I, GARCIAVERDU E, LOPEZ-PIREZ P, LLORENTE-CORTIJO IM et al. Current perspectives on the crosstalk between lung cancer stem cells and cancer-associated fibroblasts. Crit Rev Oncol Hematol 2018; 125: 102-110. https://doi.org/10.1016/j. critrevonc.2018.02.015

[37] JUNG Y, CACKOWSKI FC, YUMOTO K, DECKER AM, WANG J et al. CXCL12gamma Promotes Metastatic Castration-Resistant Prostate Cancer by Inducing Cancer Stem Cell and Neuroendocrine Phenotypes. Cancer Res 2018; 78: 2026-2039. https://doi.org/10.1158/0008-5472.CAN-172332

[38] BOESCH M, ONDER L, CHENG HW, NOVKOVIC M, MORBE $U$ et al. Interleukin 7-expressing fibroblasts promote breast cancer growth through sustenance of tumor cell stemness. Oncoimmunology 2018; 7: e1414129. https://doi. org/10.1080/2162402X.2017.1414129

[39] REN J, DING L, ZHANG D, SHI G, XU Q et al. Carcinoma-associated fibroblasts promote the stemness and chemoresistance of colorectal cancer by transferring exosomal lncRNA H19. Theranostics 2018; 8: 3932-3948. https://doi. org/10.7150/thno.25541

[40] CAZET AS, HUI MN, ELSWORTH BL, WU SZ, RODEN D et al. Targeting stromal remodeling and cancer stem cell plasticity overcomes chemoresistance in triple negative breast cancer. Nat Commun 2018; 9: 2897. https://doi.org/10.1038/ s41467-018-05220-6

[41] NAIR N, CALLE AS, ZAHRA MH, PRIETO-VILA M, OO AKK et al. A cancer stem cell model as the point of origin of cancer-associated fibroblasts in tumor microenvironment. Sci Rep 2017; 7: 6838. https://doi.org/10.1038/s41598-01707144-5

[42] ZOLLER M. CD44: can a cancer-initiating cell profit from an abundantly expressed molecule? Nat Rev Cancer 2011; 11: 254-267. https://doi.org/10.1038/nrc3023

[43] IERANO C, D'ALTERIO C, GIARRA S, NAPOLITANO M, REA G et al. CXCL12 loaded-dermal filler captures CXCR4 expressing melanoma circulating tumor cells. Cell Death Dis 2019; 10: 562. https://doi.org/10.1038/s41419-019-1796-6

[44] LI L, QI L, LIANG Z, SONG W, LIU Y et al. Transforming growth factor-beta1 induces EMT by the transactivation of epidermal growth factor signaling through HA/CD44 in lung and breast cancer cells. Int J Mol Med 2015; 36: 113 122. https://doi.org/10.3892/ijmm.2015.2222

[45] CIEPLY B, KOONTZ C, FRISCH SM. CD44S-hyaluronan interactions protect cells resulting from EMT against anoikis. Matrix Biol 2015; 48: 55-65. https://doi.org/10.1016/j. matbio.2015.04.010

[46] NIETO MA, HUANG RY, JACKSON RA, THIERY JP. Emt: 2016. Cell 2016; 166: 21-45. https://doi.org/10.1016/j. cell.2016.06.028 
[47] DEL POZO MARTIN Y, PARK D, RAMACHANDRAN A, OMBRATO L et al. Mesenchymal Cancer Cell-Stroma Crosstalk Promotes Niche Activation, Epithelial Reversion, and Metastatic Colonization. Cell Rep 2015; 13: 2456-2469. https://doi.org/10.1016/j.celrep.2015.11.025

[48] PANG X, GONG K, ZHANG X, WU S, CUI Y et al. Osteopontin as a multifaceted driver of bone metastasis and drug resistance. Pharmacol Res 2019; 144: 235-244. https://doi. org/10.1016/j.phrs.2019.04.030

[49] RAO G, WANG H, LI B, HUANG L, XUE D et al. Reciprocal interactions between tumor-associated macrophages and CD44-positive cancer cells via osteopontin/CD44 promote tumorigenicity in colorectal cancer. Clin Cancer Res 2013; 19: 785-797. https://doi.org/10.1158/1078-0432.CCR-122788

[50] KUSUYAMA J, BANDOW K, OHNISHI T, HISADOME $\mathrm{M}$, SHIMA K et al. Osteopontin inhibits osteoblast responsiveness through the down-regulation of focal adhesion kinase mediated by the induction of low-molecular weight protein tyrosine phosphatase. Mol Biol Cell 2017; 28: 13261336. https://doi.org/10.1091/mbc.E16-10-0716

[51] NAKAMURA H, HIRAGA T, NINOMIYA T, HOSOYA A, FUJISAKI N et al. Involvement of cell-cell and cell-matrix interactions in bone destruction induced by metastatic MDAMB-231 human breast cancer cells in nude mice. J Bone Miner Metab 2008; 26: 642-647. https://doi.org/10.1007/ s00774-008-0857-1

[52] KINUGASA Y, MATSUI T, TAKAKURA N. CD44 expressed on cancer-associated fibroblasts is a functional molecule supporting the stemness and drug resistance of malignant cancer cells in the tumor microenvironment. Stem Cells 2014; 32: 145-156. https://doi.org/10.1002/stem.1556

[53] CHAO YL, SHEPARD CR, WELLS A. Breast carcinoma cells re-express E-cadherin during mesenchymal to epithelial reverting transition. Mol Cancer 2010; 9: 179. https://doi. org/10.1186/1476-4598-9-179

[54] ADORNO-CRUZ V, KIBRIA G, LIU X, DOHERTY M, JUNK DJ et al. Cancer stem cells: targeting the roots of cancer, seeds of metastasis, and sources of therapy resistance. Cancer Res 2015; 75: 924-929. https://doi.org/10.1158/00085472.CAN-14-3225

[55] KAHLERT C, KALLURI R. Exosomes in tumor microenvironment influence cancer progression and metastasis. J Mol Med (Berl) 2013; 91: 431-437. https://doi.org/10.1007/ s00109-013-1020-6

[56] WEBBER J, STEADMAN R, MASON MD, TABI Z, CLAYTON A. Cancer exosomes trigger fibroblast to myofibroblast differentiation. Cancer Res 2010; 70: 9621-9630. https://doi. org/10.1158/0008-5472.CAN-10-1722

[57] HOSHINO A, COSTA-SILVA B, SHEN TL, RODRIGUES G, HASHIMOTO A et al. Tumour exosome integrins determine organotropic metastasis. Nature 2015; 527: 329-335.

[58] ZHAO Y, BACHELIER R, TREILLEUX I, PUJUGUET P, PEYRUCHAUD $O$ et al. Tumor $\alpha v \beta 3$ Integrin Is a Therapeutic Target for Breast Cancer Bone Metastases. Cancer Research 2007; 67: 5821-5830. https://doi.org/10.1158/00085472.CAN-06-4499
[59] BHOME R, GOH RW, BULLOCK MD, PILLAR N, THIRDBOROUGH SM et al. Exosomal microRNAs derived from colorectal cancer-associated fibroblasts: role in driving cancer progression. Aging (Albany NY) 2017; 9: 2666-2694. https://doi.org/10.18632/aging.101355

[60] WANG H, WEI H, WANG J, LI L, CHEN A et al. MicroRNA-181d-5p-Containing Exosomes Derived from CAFs Promote EMT by Regulating CDX2/HOXA5 in Breast Cancer. Mol Ther Nucleic Acids 2019; 19: 654-667. https://doi. org/10.1016/j.omtn.2019.11.024

[61] KONG J, TIAN H, ZHANG F, ZHANG Z, LI J et al. Extracellular vesicles of carcinoma-associated fibroblasts creates a pre-metastatic niche in the lung through activating fibroblasts. Molecular cancer 2019; 18: 175-175. https://doi. org/10.1186/s12943-019-1101-4

[62] LI YY, TAO YW, GAO S, LI P, ZHENG JM et al. Cancerassociated fibroblasts contribute to oral cancer cells proliferation and metastasis via exosome-mediated paracrine miR-34a-5p. EBioMedicine 2018; 36: 209-220. https://doi. org/10.1016/j.ebiom.2018.09.006

[63] QIN X, GUO H, WANG X, ZHU X, YAN M et al. Exosomal miR-196a derived from cancer-associated fibroblasts confers cisplatin resistance in head and neck cancer through targeting CDKN1B and ING5. Genome Biol 2019; 20: 12. https:// doi.org/10.1186/s13059-018-1604-0

[64] RICHARDS KE, ZELENIAK AE, FISHEL ML, WU J, LITTLEPAGE LE et al. Cancer-associated fibroblast exosomes regulate survival and proliferation of pancreatic cancer cells. Oncogene 2017; 36: 1770-1778. https://doi.org/10.1038/ onc. 2016.353

[65] ZHAO H, YANG L, BADDOUR J, ACHREJA A, BERNARD V et al. Tumor microenvironment derived exosomes pleiotropically modulate cancer cell metabolism. Elife 2016; 5: e10250-e10250. https://doi.org/10.7554/eLife.10250

[66] ESPOSITO M, GUISE T, KANG Y. The Biology of Bone Metastasis. Cold Spring Harb Perspect Med 2018; 8: a031252. https://doi.org/10.1101/cshperspect.a031252

[67] CROSET M, SANTINI D, IULIANI M, FIORAMONTI M, ZOCCOLI A et al. MicroRNAs and bone metastasis: a new challenge. Molecules 2014; 19: 10115-10128. https://doi. org/10.3390/molecules190710115

[68] WEIDLE UH, BIRZELE F, KOLLMORGEN G, RÜGER R. Molecular Mechanisms of Bone Metastasis. Cancer Genomics Proteomics 2016; 13: 1-12.

[69] CROSET M, PANTANO F, KAN CWS, BONNELYE E, DESCOTES $F$ et al. miRNA-30 Family Members Inhibit Breast Cancer Invasion, Osteomimicry, and Bone Destruction by Directly Targeting Multiple Bone Metastasis-Associated Genes. Cancer Res 2018; 78: 5259-5273. https://doi. org/10.1158/0008-5472.CAN-17-3058

[70] WU X, TAO P, ZHOU Q, LI J, YU Z et al. IL-6 secreted by cancer-associated fibroblasts promotes epithelial-mesenchymal transition and metastasis of gastric cancer via JAK2/ STAT3 signaling pathway. Oncotarget 2017; 8: 20741-20750. https://doi.org/10.18632/oncotarget.15119 
[71] YAN W, WU X, ZHOU W, FONG MY, CAO M et al. Cancercell-secreted exosomal miR-105 promotes tumour growth through the MYC-dependent metabolic reprogramming of stromal cells. Nat Cell Biol 2018; 20: 597-609. https://doi. org/10.1038/s41556-018-0083-6

[72] GUO L, ZHU Y, LI L, ZHOU S, YIN G et al. Breast cancer cell-derived exosomal miR-20a-5p promotes the proliferation and differentiation of osteoclasts by targeting SRCIN1. Cancer Med 2019; 8: 5687-5701. https://doi.org/10.1002/ cam 4.2454

[73] LAZAR I, CLEMENT E, ATTANE C, MULLER C, NIETO L. A new role for extracellular vesicles: how small vesicles can feed tumors' big appetite. J Lipid Res 2018; 59: 1793 1804. https://doi.org/10.1194/jlr.R083725

[74] BIGAGLI E, CINCI L, D’AMBROSIO M, LUCERI C. Transcriptomic Characterization, Chemosensitivity and Regulatory Effects of Exosomes in Spontaneous EMT/MET Transitions of Breast Cancer Cells. Cancer Genomics Proteomics 2019; 16: 163-173. https://doi.org/10.21873/cgp.20122

[75] LIN Q, CHEN X, MENG F, OGAWA K, LI M et al. ASPHnotch Axis guided Exosomal delivery of Prometastatic Secretome renders breast Cancer multi-organ metastasis. Mol Cancer 2019; 18: 156. https://doi.org/10.1186/s12943-0191077-0

[76] MAJI S, CHAUDHARY P, AKOPOVA I, NGUYEN PM, HARE RJ et al. Exosomal Annexin II Promotes Angiogenesis and Breast Cancer Metastasis. Mol Cancer Res 2017; 15: 93-105. https://doi.org/10.1158/1541-7786.MCR-16-0163

[77] LOKMAN NA, WEEN MP, OEHLER MK, RICCIARDELLI C. The role of annexin A2 in tumorigenesis and cancer progression. Cancer Microenviron 2011; 4: 199-208. https://doi. org/10.1007/s12307-011-0064-9

[78] NOY R, POLLARD JW. Tumor-associated macrophages: from mechanisms to therapy. Immunity $2014 ; 41$ : 49-61. https://doi.org/10.1016/j.immuni.2014.06.010

[79] BISWAS S, MANDAL G, ROY CHOWDHURY S, PUROHIT S, PAYNE KK et al. Exosomes Produced by Mesenchymal Stem Cells Drive Differentiation of Myeloid Cells into Immunosuppressive M2-Polarized Macrophages in Breast Cancer. J Immunol 2019; 203: 3447-3460. https://doi. org/10.4049/jimmunol.1900692
[80] YU S, LIU C, SU K, WANG J, LIU Y et al. Tumor exosomes inhibit differentiation of bone marrow dendritic cells. J Immunol 2007; 178: 6867-6875. https://doi.org/10.4049/jimmunol.178.11.6867

[81] VRACAR TC, ZUO J, PARK J, AZER D, MIKHAEL C et al. Enoxacin and bis-enoxacin stimulate $4 \mathrm{~T} 1$ murine breast cancer cells to release extracellular vesicles that inhibit osteoclastogenesis. Sci Rep 2018; 8: 16182. https://doi.org/10.1038/ s41598-018-34698-9

[82] LI D, LIU J, GUO B, LIANG C, DANG L et al. Osteoclast-derived exosomal miR-214-3p inhibits osteoblastic bone formation. Nat Commun 2016; 7: 10872. https://doi. org/10.1038/ncomms 10872

[83] LIU J, LI D, DANG L, LIANG C, GUO B et al. Osteoclastic miR-214 targets TRAF3 to contribute to osteolytic bone metastasis of breast cancer. Sci Rep 2017; 7: 40487. https://doi. org/10.1038/srep40487

[84] LIU X, CAO M, PALOMARES M, WU X, LI A et al. Metastatic breast cancer cells overexpress and secrete miR-218 to regulate type I collagen deposition by osteoblasts. Breast Cancer Res 2018; 20: 127. https://doi.org/10.1186/s13058018-1059-y

[85] YU J, LEI R, ZHUANG X, LI X, LI G et al. MicroRNA-182 targets SMAD7 to potentiate TGFbeta-induced epithelialmesenchymal transition and metastasis of cancer cells. Nat Commun 2016; 7: 13884. https://doi.org/10.1038/ncomms 13884

[86] NI X, XIA T, ZHAO Y, ZHOU W, WU N et al. Downregulation of miR-106b induced breast cancer cell invasion and motility in association with overexpression of matrix metalloproteinase 2. Cancer Sci 2014; 105: 18-25. https://doi. org/10.1111/cas.12309

[87] HAN Z, ZHAN R, CHEN S, DENG J, SHI J et al. miR-181b/ Oncostatin $\mathrm{m}$ axis inhibits prostate cancer bone metastasis via modulating osteoclast differentiation. J Cell Biochem 2020; 121: 1664-1674. https://doi.org/10.1002/jcb.29401 